\title{
Black holes with a cloud of strings in pure Lovelock gravity
}

\author{
J. M. Toledo ${ }^{\mathrm{a}}$, V. B. Bezerra ${ }^{\mathrm{b}}$ \\ Departamento de Física, CCEN, Universidade Federal da Paraíba, Caixa Postal 5008, João Pessoa, PB, Brazil
}

Received: 28 December 2018 / Accepted: 24 January 2019 / Published online: 6 February 2019

(C) The Author(s) 2019

\begin{abstract}
In this paper, we will obtain the solution corresponding to a static spherically symmetric black hole with a cloud of strings in a special class of higher curvature gravity, namely, pure Lovelock gravity. Some aspects of the thermodynamics of this black hole will be investigated, with special emphasis on the behavior of the entropy, Hawking temperature and heat capacity. The difference between these quantities and the ones corresponding the Einstein gravity is pointed out.
\end{abstract}

\section{Introduction}

The Einstein gravity is indubitable the theory that describes the gravitational phenomena at large distances. On the opposite limit, namely, at small distances, we expect that some corrections should be done in order to describe gravity appropriately. On the other hand, Einstein gravity is a non renormalizable theory in 4-dimensions, but higher derivative theories of gravity can be renormalizable in this condition [1]. Thus, we have at least one good reason to study these theories. Among the higher derivative gravity approaches, Lovelock gravity [2] is the most natural extension of Einstein gravity, where the fundamental field is the metric. It satisfies the criteria of general covariance, gives second order differential field equations and reduces to Einstein gravity in four dimensions. Additionally, Lovelock gravity is free of ghosts at a linear level [3].

Lovelock gravity is described by an action given by a homogeneous polynomial of degree $N$ in the Riemann curvature tensor, which yields, curiously, to a field equation which is a differential equation of second order in the metric, a feature of Einstein gravity, which is preserved in this extension, but it is not preserved in no other extension of Einstein gravity to dimensions other than four. Now, let us consider another

\footnotetext{
a e-mail: jefferson.m.toledo@gmail.com

be-mail: valdir@fisica.ufpb.br
}

feature of Einstein gravity in $D=3$ dimensions $((2+1)$ dimensional spacetime), namely, the kinematic property of Einstein gravity in this dimension. In fact, the generalization of this property to odd dimensions demands the necessity to formulate an extension of Einstein gravity to higher dimensions due to the fact that Einstein gravity is kinematic only in $(2+1)$-dimensions [4]. This is achieved in the framework of pure Lovelock gravity, whose action is constructed from the Lovelock gravity polynomials by taking just a single $\mathrm{Nth}$ order term. In this context, gravity has the kinematical property for all odd dimensions, $D=2 \mathrm{n}+1[4,5]$. Pure Lovelock gravity also preserves the existence of bound orbits around a static black hole as in Einstein gravity [6,7].

Black holes are very important and fascinating structures which appear in any theory of gravity. The understanding of their physics could provide some insight with respect to the formulation of a possible quantum theory of gravity. In view of these points and many others, there has been during last decades interest in these structures, especially, more recently, in the context of higher dimensions. Thus, there are good reasons to obtain black hole solutions in different theories of gravity and study their physical behavior. Vacuum solutions describing black holes in Lovelock gravity have been studied and their physical characteristics were investigated [3, 8-28]. In special, some exact solutions describing black holes were obtained in the framework of Lovelock gravity $[3,9,25,29-$ $31]$, when a cloud of strings is taken into account [19,20,32]. Others studies concerning the scenario with a cloud of strings include the analysis of the thermodynamical properties [33] and the tensor quasinormal modes [34].

In the context of the string theory, the building blocks of nature are one-dimensional objects, and not particles, which are zero-dimensional objects. In the framework of gravity, we can consider a cloud of strings as a one-dimensional ana$\log$ of a cloud of dust. The first investigations concerning a cloud of strings as a source of a gravitational field were performed by Letelier [35], who obtained a generalization of the Schwarzschild solution corresponding to a black hole 
surrounded by a spherically symmetric cloud of strings. This generalization is in the sense that the obtained metric corresponds effectively to the geometry of Schwarzschild from the local point of view, but with a solid deficit angle. Using the same formalism to construct this solution, Letelier obtained some other interesting results [36,37]. Thus, let us assume that these strings are fundamental objects in gravitational theory. Therefore, we can consider their extension to gravitational theories that go beyond Einstein's gravity, as for example Lovelock gravity [2] which we are interested in this paper, and, more specifically, pure Lovelock gravity.

Therefore, it seems to be important to investigate the behavior of black holes with a cloud of strings in the context of Lovelock gravity, more specifically pure Lovelock gravity, in order to analyze how the effect of higher-order curvature corrections change the physics of these objects.

The black hole thermodynamics is a very interesting and updated topic which can give us some insights on different aspects of black holes physics [38-40]. In this context, there is a very interesting result connected to the phenomenon of emission of radiation with a black body spectrum, termed Hawking radiation [41]. The studies related to different aspects of the black hole thermodynamics go back to the 1970's and, since then, a lot of investigations have been done with very important and interesting results [42-44].

It is the purpose of this paper to obtain the exact solution corresponding to a static and spherically symmetric black hole with a cloud of string in pure Lovelock gravity and explicitly show the effect of the cloud of strings in this context. Thus, we analyze some aspects of the thermodynamics of this black hole through the calculation of entropy, Hawking temperature and heat capacity. We will also analyze some aspects connected with the emission of scalar particles (Hawking radiation). We highlight the results in the odd and even critical dimensions in pure Lovelock gravity, which are described, respectively, by the relations $D=2 \mathrm{n}+1$ and $D=$ $2 \mathrm{n}+2$, where $n$ is the $n$th order of Lovelock polynomial.

This paper is organized as follows. In Sect. 2, we present a brief review of the Lovelock theory and, more specifically, the pure Lovelock gravity. We perform a short review related to a cloud of strings in D-dimensional Einstein gravity in Sect. 3. In Sect. 4, we obtain the static black hole solution with a cloud of strings in pure Lovelock gravity. Section 5 is devoted to the black hole thermodynamics and, in Sect. 6, we study the Hawking radiation. Finally, in Sect. 7, we present the concluding remarks. We will adopt units for which $G=$ $\mathrm{c}=1$ and the metric signature $(+,-,-,-)$.

\section{Pure Lovelock gravity}

Lovelock gravity represents a generalization of Einstein gravity to dimensions other than four. It was proposed in the 1970's by Lovelock [2] and corresponds to the unique exten- sion of Einstein gravity to higher dimensions that preserves the order of derivatives in the field equation with respect to the metric. Additionally, it is connected with string theory, from which it can be obtained in the low energy limit [45], and, thus, providing relativistic corrections to the action of Einstein gravity, namely, Einstein-Hilbert action.

In Lovelock gravity in D-dimensions, the action is given by $[16,21]$ :

$\mathscr{I}=\mathscr{I}_{L}+\mathscr{I}_{M}=\frac{1}{2} \int_{\mathscr{M}} d x^{D} \sqrt{-g} R+\mathscr{I}_{M}$,

where $\mathscr{I}_{M}$ is the action related to the matter fields and

$R=\sum_{p=0}^{n} \alpha_{p} R^{(p)}$

with $\alpha_{p}$ being the coupling constants, $n=(D-1) / 2$ for odd dimensions, $n=(D-2) / 2$ in even dimensions and

$R^{(p)}=\frac{1}{2^{p}} \delta_{\alpha_{1} \ldots \alpha_{p} \beta_{1} \ldots \beta_{p}}^{\mu_{1} \ldots \mu_{p} \nu_{1} \ldots v_{p}} R_{\mu_{1} \nu_{1}}^{\alpha_{1} \beta_{1}} \ldots R_{\mu_{p} v_{p}}^{\alpha_{p} \beta_{p}}$

The generalized Kronecker delta, $\delta_{\alpha_{1} \ldots \alpha_{p} \beta_{1} \ldots \beta_{p}}^{\mu_{1} \ldots \mu_{p} \nu_{1} \ldots v_{p}}$, is totally antisymmetric by permutation of any index and could be obtained by

$\delta_{\alpha_{1} \ldots \alpha_{p}}^{\mu_{1} \ldots \mu_{p}}=p ! \delta_{\left[\alpha_{1} \ldots \alpha_{p}\right]}^{\mu_{1} \ldots \mu_{p}}$.

For $R^{(1)}=R$ and $\alpha_{p}=0$, for $p \neq 1$, we reobtain the Einstein-Hilbert action. In the particular case where $\alpha_{p}=0$, for $p>3$, we get the Lagrangian of Gauss-Bonnet gravity. We can assume that the parameter $\alpha_{0}$ can be given by $\alpha_{0}=-2 \Lambda$, where $\Lambda$ is the cosmological constant. Taking the variation of the Lovelock action with respect to the metric, we find the generalized Einstein equation [46]

$$
\begin{aligned}
& -\frac{\alpha_{0}}{2} g_{\mu \nu}+\sum_{p=0}^{n} \alpha_{p} G_{\mu \nu}^{(p)} \\
& =-\frac{\alpha_{0}}{2} g_{\mu \nu}+\sum_{p=0}^{n} \alpha_{p}\left[p\left(R_{\mu \nu}^{(p)}-\frac{1}{2} R^{(p)} g_{\mu \nu}\right)\right] \\
& =T_{\mu \nu}
\end{aligned}
$$

where $R_{\mu \nu}^{(p)}=g^{\sigma \rho} R_{\mu \nu \sigma \rho}^{(p)}, R^{(p)}=g^{\mu \nu} R_{\mu \nu}^{(p)}, T_{\mu \nu}$ is the energy-momentum tensor given by the following relations $[5,47]$ 
$R_{\mu \nu \sigma \rho}^{(p)}=F_{\mu \nu \sigma \rho}^{(p)}-\frac{p-1}{p(d-1)(d-2)} F^{(n)}\left(g_{\mu \sigma} g_{\nu \rho}-g_{\mu \rho} g_{\nu \sigma}\right)$,

$F_{\mu \nu \sigma \rho}^{(p)}=Q_{\mu \nu}{ }^{\alpha \beta} R_{\sigma \rho \alpha \beta}$,

$Q^{\mu \nu}{ }_{\alpha \beta}=\delta_{\alpha \beta \alpha_{1} \beta_{1} \ldots \alpha_{n} \beta_{n}}^{\mu \nu \mu_{1} v_{1} \ldots \mu_{n} v_{n}} R_{\alpha_{1} \beta_{1}}^{\mu_{1} v_{1}} \ldots R_{\alpha_{n} \beta_{n}}{ }^{\mu_{n} v_{n}}$,

$Q_{; \rho}^{\mu \nu \sigma \rho}=0$,

$T_{\mu \nu}=\frac{2}{\sqrt{-g}} \frac{\delta \mathscr{I}_{M}}{\delta g^{\mu \nu}}$.

The Lovelock gravity is named pure when we take a fixed value of $p$ in the equations above, which means that the homogeneous polynomial of the action contains just one term of order $p$ in the Riemann curvature tensor. Thus, if we consider $p=n$, we write the field equation in pure Lovelock gravity in order $n$ as

$$
\begin{aligned}
& -\frac{\alpha_{0}}{2} g_{\mu \nu}+\alpha_{n} G_{\mu \nu}^{(n)} \\
& =-\frac{\alpha_{0}}{2} g_{\mu \nu}+\alpha_{n}\left[n\left(R_{\mu \nu}^{(n)}-\frac{1}{2} R^{(n)} g_{\mu \nu}\right)\right]=T_{\mu \nu} .
\end{aligned}
$$

\section{Cloud of strings}

The first studies concerning a formalism to treat gravity with a cloud of strings as a source, in the framework of Einstein gravity, was presented by Letelier [35], who obtained a generalization of the Schwarzschild solution corresponding to a black hole surrounded by a spherically symmetric cloud of strings, whose energy-momentum tensor is given by

$$
\begin{aligned}
T_{t}^{t} & =T_{r}^{r}=\rho_{c}=\frac{a}{r^{2}} \\
T_{\theta}^{\theta} & =T_{\phi}^{\phi}=0,
\end{aligned}
$$

where $\rho_{c}$ is the energy density of the cloud and $a$ is an integration constant associated with the presence of the cloud of strings. The stress-energy tensor for this case has the form of Eq. (8) due to the spherical symmetry of the configuration [35]. Solving Einstein's equation taking into account the source given by Eq. (8), Letelier found that [35] the spacetime metric is given by

$$
\begin{aligned}
d s^{2}= & \left(1-a-\frac{2 M}{r}\right) d t^{2} \\
& -\left(1-a-\frac{2 M}{r}\right)^{-1} d r^{2}-r^{2} d \Omega^{2} .
\end{aligned}
$$

On the other hand, the solution corresponding to a black hole with a cloud of strings, in a D-dimensional spacetime, is given by the general form [20]

$d s^{2}=f(r) d t^{2}-\frac{1}{f(r)} d r^{2}-r^{2} d \Omega_{D-2}^{2}$.
In this case, the energy-momentum tensor corresponding to a cloud of strings, spherically symmetric, is given by [20, 32]

$T_{\nu}^{\mu}=\frac{a}{r^{D-2}}[1,1,0, \ldots, 0]$,

and

$f(r)=1-\frac{\mu}{r^{D-3}}-\frac{2 a}{(D-2) r^{D-4}}$,

where

$\mu=\frac{16 \pi M}{(D-2) \Sigma_{D-2}}$,

with

$\Sigma_{D-2}=\frac{2 \pi^{(D-1) / 2}}{\Gamma[(D-1) / 2]}$,

being the volume of the D-sphere.

Therefore, the metric corresponding to a spherically symmetric and static black hole with a cloud of strings in a D dimensional spacetime is given by Eq. (10) combined with Eqs. (12)-(14).

\section{Black hole with a cloud of strings in pure Lovelock gravity}

In $D$ dimensions, consider a spacetime metric written like Eq. (10). Let us assume that $[16,25,31]$

$f(r)=\kappa-r^{2} F(r)$,

where $F(r)$ is a solution of the polynomial equation

$$
\begin{aligned}
P(F) & =\sum_{p=0}^{m} \widetilde{\alpha}_{p} F^{p} \\
& =\frac{16 \pi M}{(D-2) \Sigma_{D-2} r^{D-1}}-\frac{2 a}{(D-2) r^{D-2}},
\end{aligned}
$$

with

$\widetilde{\alpha}_{0}=\frac{\alpha_{0}}{(D-1)(D-2)}$,

$\widetilde{\alpha}_{1}=1$,

$\widetilde{\alpha}_{p}=\prod_{i=3}^{2 p}(D-i) \alpha_{p}, \quad p>1$.

In pure Lovelock gravity, for $p=n$ [23], Eq. (16) can be written as

$\tilde{\alpha}_{n} F^{n}=\frac{16 \pi M}{(D-2) \Sigma_{D-2} r^{D-1}}-\frac{2 a}{(D-2) r^{D-2}}$, 
Taking, for simplicity, $\tilde{\alpha}_{n}=\alpha^{2 n-2}$ [23], where $\alpha$ is a scale length, we get

$F(r)=\left\{\begin{array}{l} \pm \frac{1}{\alpha^{2-2 / n}}\left[\frac{16 \pi M}{(D-2) \Sigma_{D-2} r^{D-2 n+1}}-\frac{2 a}{(D-2) r^{D-2 n}}\right]^{\frac{1}{n}}, n \text { even } \\ \frac{\operatorname{sign}(x)}{\alpha^{2-2 / n}}\left[\frac{16 \pi M}{(D-2) \Sigma_{D-2} r^{D-2 n+1}}-\frac{2 a}{(D-2) r^{D-2 n}}\right]^{\frac{1}{n}}, n \text { odd }\end{array}\right.$

where $x=\frac{2 M}{(D-2) \Sigma_{D-2} r^{D-2 n+1}}-\frac{2 a}{(D-2) r^{D-2 n}}$. From Eqs. (15) and (19), we conclude that

$f(r)=\left\{\begin{array}{l}\kappa \mp \frac{1}{\alpha^{2-2 / n}}\left[\frac{16 \pi M}{(D-2) \Sigma_{D-2} r^{D-2 n-1}}-\frac{2 a}{(D-2) r^{D-2 n-2}}\right]^{\frac{1}{n}}, n \text { even } \\ \kappa-\frac{\operatorname{sign}(x)}{\alpha^{2-2 / n}}\left[\frac{16 \pi M}{(D-2) \Sigma_{D-2} r^{D-2 n-1}}-\frac{2 a}{(D-2) r^{D-2 n-2}}\right]^{\frac{1}{n}}, n \text { odd. }\end{array}\right.$

In the Appendix A, we will show how to obtain the solution given by Eq. (20) using another procedure. The obtained metric has an interesting property [23], which is the following: if $r_{x}$ is such that $x=0$, the Kretschmann scalar, defined by

$$
\begin{aligned}
R_{\mu \nu \sigma \rho} R^{\mu \nu \sigma \rho}= & f^{\prime \prime 2}(r)+2(D-2) \frac{f^{\prime}(r)}{r^{2}} \\
& +2(D-2)(D-3) \frac{f(r)^{2}}{r^{4}},
\end{aligned}
$$

diverges. Thus, we will take into account only values of $r$ such that $r>r_{x}$. The Kretschmann scalar also diverges for $r=0$ and, therefore, this point is a physical singularity. If we take $\kappa=1$ and $x>0$, we get

$$
\begin{aligned}
f(r)= & 1-\frac{1}{\alpha^{2-2 / n}}\left[\frac{16 \pi M}{(D-2) \Sigma_{D-2} r^{D-2 n-1}}\right. \\
& \left.-\frac{2 a}{(D-2) r^{D-2 n-2}}\right]^{\frac{1}{n}} .
\end{aligned}
$$

Thus, if $n=1$, we obtain the known solution for a black hole in $D$ dimensions surrounded by cloud of strings:

$f(r)=1-\frac{\mu}{r^{D-3}}-\frac{2 a}{(D-2) r^{D-4}}$,

which, in 4 dimensions reduces to

$f(r)=1-a-\frac{2 M}{r}$.

Now, let us verify the properties of the proposed solution in the odd and even critical dimensions, respectively, $D=2 \mathrm{n}$ +1 and $D=2 \mathrm{n}+2$, where $n$ is the $n$th order of Lovelock polynomial in the action. In the odd critical dimensions, Eq. (20) can be written as

$f_{o}(r)=1-\frac{1}{\alpha^{2-2 / n}}\left[\frac{8 M \Gamma(n)}{(2 n-1) \pi^{n-1}}-\frac{2 a}{(2 n-1) r}\right]^{\frac{1}{n}}$.
On the other hand, in even critical dimensions, we get

$f_{e}(r)=1-\frac{1}{\alpha^{2-2 / n}}\left[\frac{4 M \Gamma(n+1 / 2)}{n \pi^{n-1 / 2} r}-\frac{a}{n}\right]^{\frac{1}{n}}$.

Observe that, in the critical dimensions, the form of the Eqs. (25) and (26) maintains a similarity with Einstein theory. In the even critical dimensions, the cloud of strings adds a term that does not depends on the radial coordinate $r$.

The black hole horizons are defined by the radial coordinate $r_{h}$, which is obtained from the equality

$f\left(r_{h}\right)=0$.

In odd critical dimensions, we get

$r_{h}^{(o)}=\left[\frac{4 M \Gamma(n)}{a \pi^{n-1}}-\frac{\alpha^{2 n-2}(2 n-1)}{2 a}\right]^{-1}$.

Thus, in odd critical dimensions, the black hole will have horizons if $\alpha^{2 n-2}<\frac{8 M \Gamma(n)}{\pi^{n-1}(2 n-1)}$. In even critical dimensions, the black hole horizons will be given by

$r_{h}^{(e)}=\frac{4 M \Gamma(n+1 / 2)}{\pi^{n-1 / 2}\left(n \alpha^{2 n-2}+a\right)}$.

For even critical dimensions, if $a>-n \alpha^{2 n-2}$, the black holes will always have horizons.

In Figs. 1 and 2, we show the behaviors of the functions $f_{o}(r)$ and $f_{e}(r)$ in the Einstein gravity $(n=1)$, in the GaussBonnet gravity $(n=2)$ and in pure Lovelock gravity with $n=3$.

\section{Black hole thermodynamics}

In this section, we study the black hole thermodynamics, in order to compare the properties of the obtained solution in the critical dimensions.

In Appendix B, we add the cosmological constant in the metric under consideration and also perform the thermodynamical analysis.

In the black hole horizon, $f\left(r_{h}\right)=0$. Thus, the black hole mass can be written as

$M=\frac{(D-2) \Sigma_{D-2}}{16 \pi}\left[\alpha^{2 n-2} r_{h}^{D-2 n-1}+\frac{2 a}{D-2} r_{h}\right]$.

Note that the cloud of string term do not depend on the order of the pure Lovelock gravity, $n$, and the first term in the equation depends on $r_{h}$ in the same way as is presented in the literature [23]. In the odd and even critical dimensions, we get, respectively,

$M_{o}=\frac{(2 n-1) \pi^{n-1}}{8 \Gamma(n)}\left[\alpha^{2 n-2}+\frac{2 a}{2 n-1} r_{h}\right]$,

$M_{e}=\frac{n \pi^{n-1 / 2} r_{h}}{4 \Gamma(n+1 / 2)}\left[\alpha^{2 n-2}+\frac{a}{n}\right]$. 

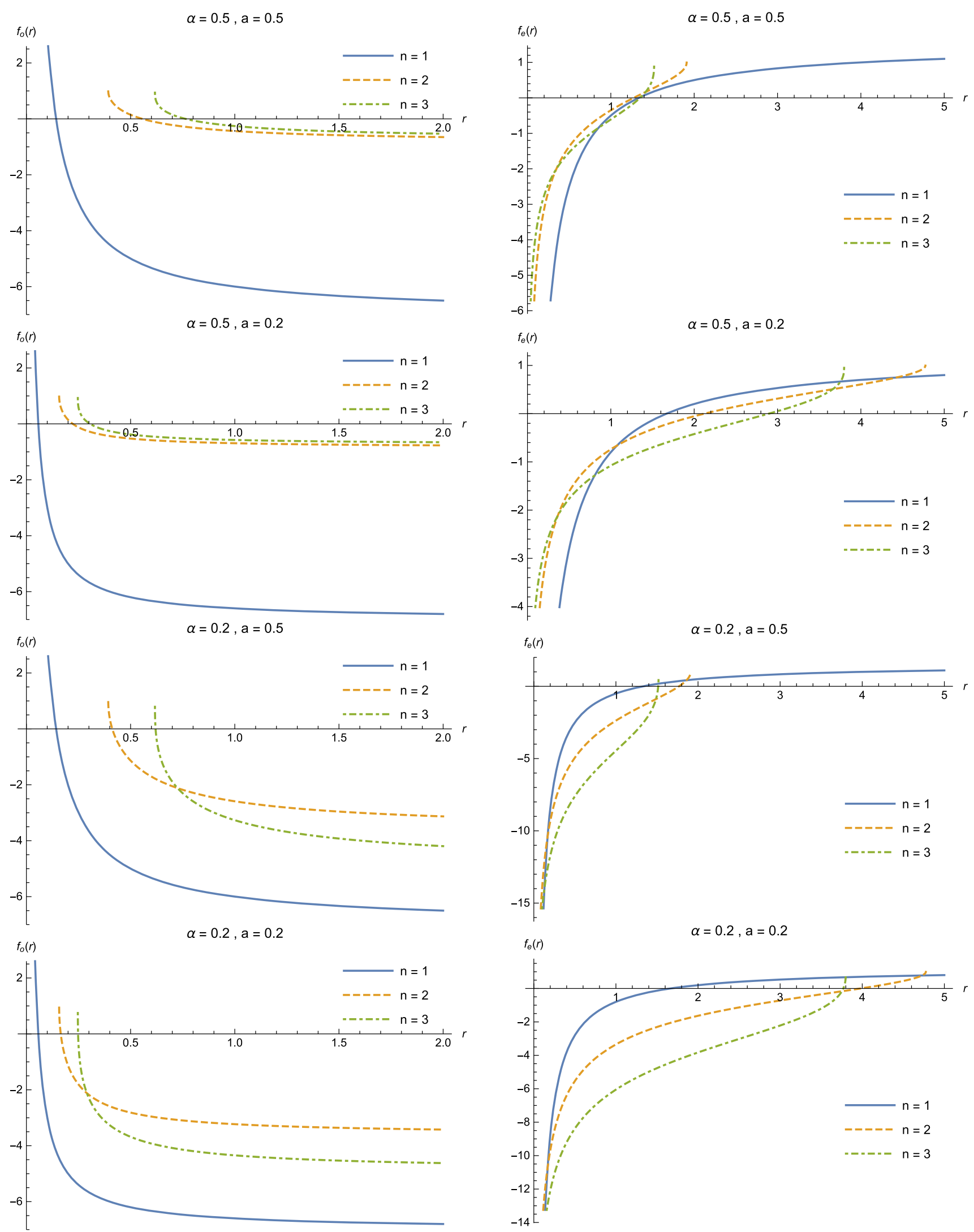

Fig. 1 The function $f_{o}(r)$, for $M=1$

Fig. 2 The function $f_{o}(r)$, for $M=1$ 
In both critical dimensions, the black hole mass parameter has a linear dependence on $r_{h}$ and, when the order of pure Lovelock gravity is increased, the mass decreases.

The Hawking temperature of the black hole is calculated from

$T=\left.\frac{1}{4 \pi} \frac{d f(r)}{d r}\right|_{r=r_{h}}$.

Thus, we get

$T=\frac{\alpha^{2-2 n}}{4 \pi n}\left[\frac{\alpha^{2 n-2}(D-2 n-1)}{r_{h}}+\frac{2 a}{(D-2) r_{h}^{D-2 n-1}}\right]$,

which, in critical dimensions can be written as

$$
\begin{aligned}
T_{o} & =\frac{\alpha^{2-2 n} a}{2 \pi(2 n-1) n}, \\
T_{e} & =\frac{1}{4 \pi n r_{h}}\left[1+\frac{a \alpha^{2-2 n}}{n}\right] .
\end{aligned}
$$

Thus, considering the odd critical dimensions, we see that the Hawking temperature is constant with respect to the horizon radius and decreases when $n$ increases.

Using the First Law of the black hole thermodynamics, $d M=T d S$, the black hole entropy can be calculated by [32]

$$
\begin{aligned}
S & =\int \frac{d M}{T}=\int T^{-1} \frac{\partial M}{\partial r_{h}} d r_{h} \\
& =\frac{n(D-2) \Sigma_{D-2}}{4(D-2 n) \alpha^{2-2 n}} r_{h}^{D-2 n},
\end{aligned}
$$

It is worth observing that the integration in the previous equation was done in the interval from 0 to $r_{h}$, which implies that the entropy vanishes at $r_{h}=0$. Note that the entropy $S$ depends on the cloud of string parameter, $a$, only indirectly through the horizon coordinate $r_{h}$. Besides that, for $D>2 n$, the entropy always assumes positive values. In the critical dimensions, the entropy assumes the values

$S_{o}=\frac{n(2 n+1) \pi^{n}}{2 \alpha^{2-2 n} \Gamma(n)} r_{h}$,

$S_{e}=\frac{n^{2} \pi^{n+1 / 2}}{2 \alpha^{2-2 n} \Gamma(n+1 / 2)} r_{h}^{2}$.

The area of a hypersphere in D-dimensions is given by $A_{d}=2 \pi^{(d+1) / 2} r^{n} / \Gamma[(d+1) / 2]$. Thus, in Einstein gravity in 4-dimensions, the black hole entropy can be find from the area law

$S=\pi r_{h}^{2}=\frac{A_{3}}{4}$,

Note that a similar relation cannot be obtained in the odd critical dimensions.
Finally, the black hole heat capacity is given by

$C=\frac{\partial M}{\partial T}=\frac{\partial M}{\partial r_{h}} \frac{\partial r_{h}}{\partial T}=\frac{n(D-2) \Sigma_{D-2}}{4 \alpha^{2-2 n}} \frac{C_{1}\left(r_{h}\right)}{C_{2}\left(r_{h}\right)}$,

where

$C_{1}=\alpha^{2 n-2}(D-2 n-2) r_{h}^{D-2 n-2}+\frac{2 a}{D-2}$,

$C_{2}=-\frac{\alpha^{2 n-2}(D-2 n-1)}{r_{h}^{2}}+\frac{2 a(D-2 n-1)}{(D-2) r_{h}^{D-2 n}}$.

In even critical dimensions, the heat capacity, as a function of $r_{h}$, can be written as

$C_{e}=\frac{a n^{2} \pi^{n+1 / 2}}{\Gamma(n+1 / 2)} \frac{r_{h}^{2}}{a \alpha^{2-2 n}-n}$,

which assumes finite values while it diverges in the odd critical dimensions. In Fig. 3, it is represented the behavior of $C_{e}$ for different values of the parameters $a$ and $\alpha$. We can verify that the signal of the heat capacity changes for different values of the order of the pure Lovelock gravity and the parameters under consideration. In other words, the black hole can be thermodynamically stable (when $C_{e}$ is positive) or unstable (when $C_{e}$ is negative).

\section{Hawking radiation}

Now, let us consider the phenomenon concerning the radiation of scalar particles by a black hole, which was pointed out by Hawking [40]. Taking $r_{+}$, the coordinate $r$ of the event horizon far away from the black hole, we can perform the coordinate transformation [48]

$d T=d t+\frac{1}{f(r)} \sqrt{\frac{r_{+}}{r}} d r$.

Thus, the metric given by Eq. (10) turns into

$$
\begin{aligned}
d s^{2}= & -f(r) d T^{2}+2 \sqrt{\frac{r_{+}}{r}} d T d r \\
& +\frac{r-r_{+}}{r} \frac{1}{f(r)} d r^{2}+r^{2} d \Omega_{D-2} .
\end{aligned}
$$

As can be verified, $r=r_{+}$is not a singularity of this spacetime [48]. The null radial geodesic is obtained from

$\frac{d r}{d T}=f(r)\left(1+\sqrt{\frac{r_{+}}{r}}\right)^{-1}$.

Therefore, the imaginary part of the action corresponding to the particle which crosses the horizon is given by:

$$
\begin{aligned}
\operatorname{ImZ} & =\operatorname{Im} \int_{T_{i}}^{T_{f}} L d T=\operatorname{Im} \int_{r_{i}}^{r_{f}} p_{r} d r \\
& =\operatorname{Im} \int_{r_{i}}^{r_{f}} \int_{0}^{p_{r}} d p_{r} d r,
\end{aligned}
$$



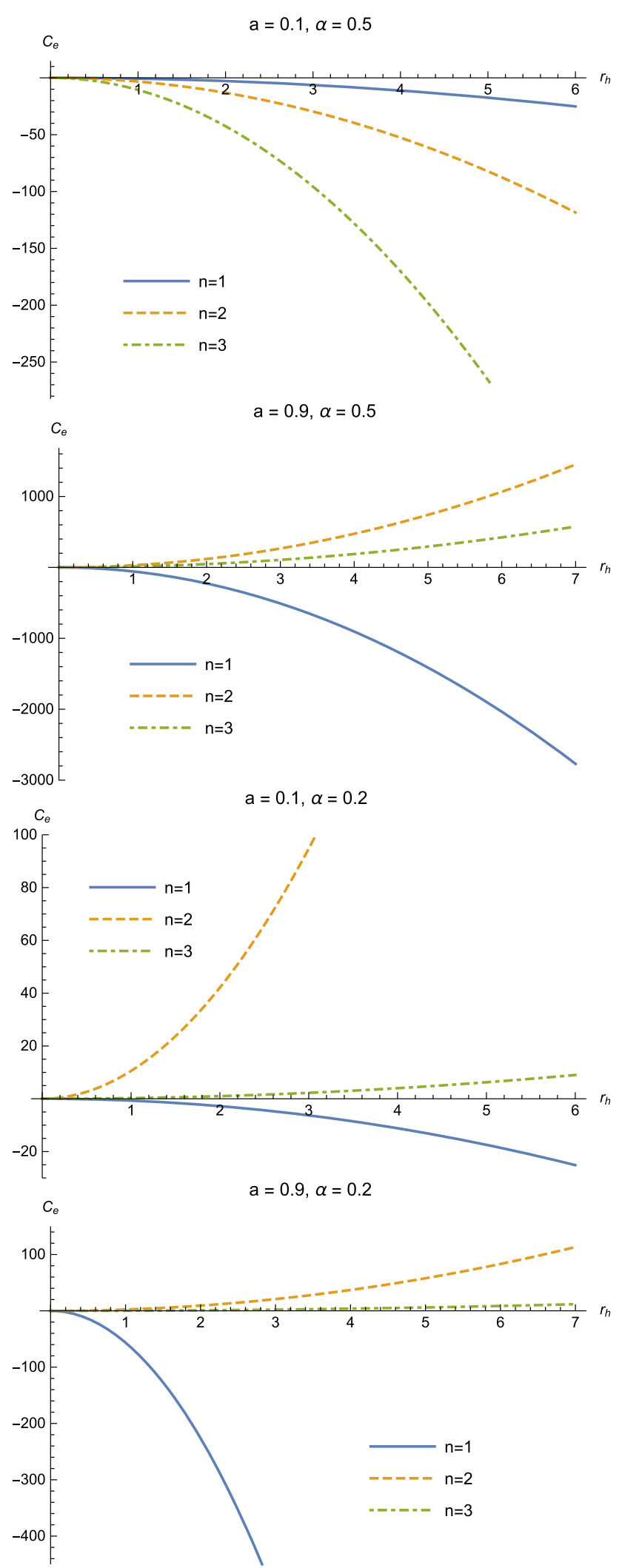

Fig. 3 The heat capacity $C_{e}$ as a function of $r_{h}$ for $M=1$ where $p_{r}$ is the canonical momentum associated with the coordinate $r$. Thus, using the relation $\dot{r}=\left.\frac{d H}{d p_{r}}\right|_{r}$ and the fact that $\left(d H_{r}\right)=d M$, we find that

$$
\begin{aligned}
\operatorname{Im} Z & =\operatorname{Im} \int_{M_{i}}^{M_{f}} \int_{r_{i}}^{r_{f}} \frac{d r}{\dot{r}} d M \\
& =\operatorname{Im} \int_{M_{i}}^{M_{f}} \int_{r_{i}}^{r_{f}} \frac{1+\sqrt{r_{+} / r}}{f(r)} d r d M,
\end{aligned}
$$

where $M_{i}=M$ is the original mass of the black hole and $M_{f}=M-\omega$ is the mass after the emission of a particle with energy $\omega$. Note that the integrand has a pole at $r=r_{+}$, and, thus, we can calculate the contour integral around that pole as

$$
\begin{aligned}
\operatorname{Im} Z & =-2 \pi \int_{M_{i}}^{M_{f}} \frac{1}{f^{\prime}\left(r_{+}\right)} d M \\
& =-2 \pi \int_{r_{+i}}^{r_{+f}} \frac{1}{f^{\prime}\left(r_{+}\right)} \frac{d M}{d r_{+}} d r_{+} .
\end{aligned}
$$

From Eqs. (15) and (30), we find

$$
\operatorname{Im} Z=-\left.\frac{n(D-2) \Sigma_{D-2}}{8(D-2 n) \alpha^{2-2 n}} r_{+}^{D-2 n}\right|_{r_{+i}} ^{r_{+f}}=-\frac{\Delta S}{2} .
$$

Using the fact that, from the WKB method, the probability that a particle emitted by the black hole experience tunneling is given by

$\Gamma \sim \exp (-2 \operatorname{ImZ})$

we find that

$\Gamma \sim \exp \left[-\frac{n(D-2) \Sigma_{D-2}}{8(D-2 n) \alpha^{2-2 n}}\left(r_{+f}^{D-2 n}-r_{+i}^{D-2 n}\right)\right]$,

or

$\Gamma \sim e^{\Delta S}$

which means that this probability is related to the change of the entropy of the black hole. Thus, it depends on the event horizon squared for even critical dimensions, similarly to what occurs in Einstein gravity.

\section{Concluding remarks}

The obtained solutions corresponding to a black hole with a cloud of strings, for critical odd and even dimensions are analogous to the ones find in the context of Einstein gravity. As to the horizons, for odd critical dimensions, they exist if some conditions are satisfied, which does not involve the parameter $a$, which codifies the presence of the cloud of strings. On the other hand, for even critical dimensions the condition that guarantees the existence of horizons involves the parameter $a$. 
Concerning the parameter of mass, for both critical dimensions, this parameter depends linearly on the radius of the horizon. It decreases when the order of the polynomial increases for both cases, namely, odd and even dimensions.

The Hawking temperature does not depend on the radius of the horizon for odd critical dimensions, while depends on this quantity for even critical dimensions.

The entropy for odd and even dimensions given by Eqs. (36) and (37) shows us that for even critical dimensions, the area law which is obtained in the context of Einstein gravity is preserved. This does not occur for odd critical dimensions.

As to the heat capacity, it diverges for odd critical dimensions and has a behavior similar to the Einstein gravity in the case of even critical dimensions.

The probability that a particle is emitted by the black hole depends on the entropy whose behavior depends on the critical dimensions, namely, even or odd. In particular, for even critical dimensions, the entropy is proportional to the horizon radius squared and, thus, it behaves similarly to what occurs in Einstein gravity.

Therefore, the thermodynamical quantities, like temperature, entropy and heat capacity behave in an appropriate way depending if the critical dimension is even or odd. In particular, for all even dimensions, entropy is proportional to the square of the horizon radius, but this behavior is not preserved for odd dimensions. As to the rate of emission of particles, this quantity has a similar behavior as compared with the one in Einstein gravity for even critical dimensions, but a different behavior for odd critical dimensions.

Acknowledgements V. B. Bezerra is partially supported by Conselho Nacional de Desenvolvimento Científico e Tecnológico (CNPq) through the research Project nr. 305835/2016-5.

Data Availability Statement This manuscript has no associated data or the data will not be deposited. [Author's comment: This paper concerns only to theoretical investigations and therefore does not contain any associated data.]

Open Access This article is distributed under the terms of the Creative Commons Attribution 4.0 International License (http://creativecomm ons.org/licenses/by/4.0/), which permits unrestricted use, distribution, and reproduction in any medium, provided you give appropriate credit to the original author(s) and the source, provide a link to the Creative Commons license, and indicate if changes were made.

Funded by SCOAP ${ }^{3}$.

\section{Appendix A: Another way to obtain the solution for a black hole surrounded by a cloud of strings in pure Love- lock gravity}

In this section, we will reobtain the solution corresponding to the black hole under consideration by directly integrating the generalized Einstein's equation in pure Lovelock gravity. This result will confirm that the solution we have working with and the method developed by Cai $[23,25]$. Let us start supposing that the metric of the black hole has the form of Eq. (10). If $f=1-F$, Dadhich [49] shown that the generalized Einstein's equation is written as

$G^{\mu}{ }_{\nu}=-\frac{D-2}{r^{D-2}}\left(r^{D-2 n-1} F^{n}\right)^{\prime}=T_{r}^{r}=T^{t}$.

where the comma denotes differentiation with respect to $r$. In the exterior region of the black hole surrounded by a cloud of strings, we can substitute Eq. (8) into Eq. (A.1), to obtain

$\left(r^{D-2 n-1} F^{n}\right)^{\prime}=-\frac{a}{D-2}$.

Integrating both sides of Eq. (A.2), we get

$F^{n}=\frac{\mu}{r^{D-2 n-1}}-\frac{a}{(D-2) r^{D-2 n-2}}$.

It is worth noting that Eq. (A.3) is similar to Eq. (18) if we make the substitutions $\mu \rightarrow \frac{2 M}{\tilde{\alpha}_{n}(D-2) \Sigma_{D-2}}$ and $a \rightarrow$ $\frac{a}{\tilde{\alpha}_{n}}$. Thus, we reobtain the solution of the black hole under consideration. If we add the cosmological constant to the gravitational background, we get [49]

$F^{n}=\Lambda_{1} r^{2 n}+\frac{\mu}{r^{D-2 n-1}}-\frac{a}{(D-2) r^{D-2 n-2}}$,

where $\Lambda_{1}=\frac{2 \Lambda}{(d-1)(d-2)}$.

\section{Appendix B: dS/AdS Black hole with a cloud of strings in pure Lovelock gravity}

In this appendix, we will deal with the black hole with a cloud of strings in pure Lovelock gravity in the background with cosmological constant $\Lambda$. Thus, we will take $\left|\alpha_{0}\right|=2 \Lambda$ into Eq. (7), and thus we obtain

$$
\begin{aligned}
- & \Lambda g_{\mu \nu}+\alpha_{n} G_{\mu \nu}^{(n)} \\
& =-\Lambda g_{\mu \nu}+\alpha_{n}\left[n\left(R_{\mu \nu}^{(n)}-\frac{1}{2} R^{(n)} g_{\mu \nu}\right)\right]=T_{\mu \nu} .
\end{aligned}
$$

According to the contents of Appendix A, the function $f(r)$ can be written as

$$
\begin{aligned}
f(r)=1- & \frac{1}{\alpha^{2-2 / n}}\left[\Lambda_{1} r^{2 n}+\frac{16 \pi M}{(D-2) \Sigma_{D-2} r^{D-2 n-1}}\right. \\
& \left.-\frac{2 a}{(D-2) r^{D-2 n-2}}\right]^{\frac{1}{n}} .
\end{aligned}
$$

In the limit $r \rightarrow \infty$, we get

$$
\begin{aligned}
f(r)=1- & \frac{1}{\alpha^{2-2 / n}}\left[\Lambda_{1}^{1 / n} r^{2}+\frac{16 \pi M}{(D-2) \Sigma_{D-2} r^{D-3}}\right. \\
& \left.-\frac{2 a}{(D-2) r^{D-4}}\right] .
\end{aligned}
$$


Thus, the asymptotic behavior of the black hole spacetime corresponds to the solution obtained in Einstein gravity, as should be expected [49].

Now, let us briefly develop the black hole thermodynamics adding the cosmological constant in the gravitational background. In this scenario, the mass parameter of the Eq. (30) will be given by

$$
\begin{aligned}
M= & \frac{(D-2) \Sigma_{D-2}}{16 \pi} \\
& {\left[\alpha^{2 n-2} r_{h}^{D-2 n-1}+\frac{2 a}{D-2} r_{h}-\Lambda r_{h}^{D-1}\right], }
\end{aligned}
$$

which has an additional term corresponding to the cosmological constant.

With respect to the Hawking temperature, we find

$$
\begin{aligned}
T=\frac{\alpha^{2-2 n}}{4 \pi n} & {\left[\frac{\alpha^{2 n-2}(D-2 n-1)}{r_{h}}\right.} \\
+ & \left.\frac{2 a}{(D-2) r_{h}^{D-2 n-1}}-(D-1) \Lambda r^{2 n-1}\right] .
\end{aligned}
$$

Thus, the entropy can be calculated from

$$
\begin{aligned}
S & =\int \frac{d M}{T}=\int T^{-1} \frac{\partial M}{\partial r_{h}} d r_{h} \\
& =\frac{n(D-2) \Sigma_{D-2}}{4(D-2 n) \alpha^{2-2 n}} r_{h}^{D-2 n} .
\end{aligned}
$$

Besides that, we can identify the cosmological constant with the thermodynamical pressure by [50]

$p=-\frac{\Lambda}{8 \pi}$.

Thus, we can write the First Law of the black hole thermodynamics

$d M=T d S+V d p$,

where

$V=\frac{\partial M}{\partial p}=\frac{(D-2) \Sigma_{D-2}}{2} r_{h}^{D-1}$.

Therefore, we can examine the thermodynamics of this new black hole by following straightforwardly what was done in the case where the cosmological constant is absent.

\section{References}

1. K. Stelle, Phys. Rev. D 16(4), 953 (1977)

2. D. Lovelock, J. Math. Phys. 12(3), 498 (1971)

3. D.G. Boulware, S. Deser, Phys. Rev. Lett. 55(24), 2656 (1985)

4. N. Dadhich, Eur. Phys. J. C 76(3), 104 (2016)

5. N. Dadhich, S.G. Ghosh, S. Jhingan, Phys. Lett. B 711(2), 196 (2012)
6. N. Dadhich, J.M. Pons, J. Math. Phys. 54(10), 102501 (2013)

7. N. Dadhich, J.M. Pons, K. Prabhu, Gen. Relativ. Gravit. 44(10), $2595(2012)$

8. B. Whitt, Phys. Rev. D 38(10), 3000 (1988)

9. J.T. Wheeler, Nucl. Phys. B 268(3-4), 737 (1986)

10. N. Dadhich, J.M. Pons, K. Prabhu, Gen. Relativ. Gravit. 45(6), 1131 (2013)

11. H. Maeda, Phys. Rev. D 73(10), 104004 (2006)

12. H. Maeda, S. Willison, S. Ray, Class. Quantum Gravity 28(16), 165005 (2011)

13. M. Nozawa, H. Maeda, Class. Quantum Gravity 23(5), 1779 (2006)

14. H. Maeda, Class. Quantum Gravity 23(6), 2155 (2006)

15. M. Dehghani, N. Farhangkhah, Phys. Rev. D 78(6), 064015 (2008)

16. S.G. Ghosh, S.D. Maharaj, D. Baboolal, T.H. Lee, Eur. Phys. J. C 78(2), 90 (2018)

17. B. Cvetković, D. Simić, Class. Quantum Gravity 35(5), 055005 (2018)

18. S.H. Mazharimousavi, M. Halilsoy, Phys. Lett. B 681(2), 190 (2009)

19. E. Herscovich, M.G. Richarte, Phys. Lett. B 689(4), 192 (2010)

20. S.G. Ghosh, U. Papnoi, S.D. Maharaj, Phys. Rev. D 90(4), 044068 (2014)

21. S.G. Ghosh, S.D. Maharaj, Phys. Rev. D 89(8), 084027 (2014)

22. S.G. Ghosh, Phys. Lett. B 704(1-2), 5 (2011)

23. R.G. Cai, N. Ohta, Phys. Rev. D 74(6), 064001 (2006)

24. D. Wiltshire, Phys. Lett. B 169(1), 36 (1986)

25. R.G. Cai, Phys. Lett. B 582(3), 237 (2004)

26. J. Grain, A. Barrau, P. Kanti, Phys. Rev. D 72(10), 104016 (2005)

27. X.O. Camanho, J.D. Edelstein, Class. Quantum Gravity 30(3), 035009 (2013)

28. C. Garraffo, G. Giribet, Mod. Phys. Lett. A 23(22), 1801 (2008)

29. R.A. Hennigar, E. Tjoa, R.B. Mann, J. High Energy Phys. 2017(2), 70 (2017)

30. R.A. Hennigar, R.B. Mann, E. Tjoa, Phys. Rev. Lett. 118(2), 021301 (2017)

31. R.C. Myers, J.Z. Simon, Phys. Rev. D 38(8), 2434 (1988)

32. T.H. Lee, D. Baboolal, S.G. Ghosh, Eur. Phys. J. C 75(7), 297 (2015)

33. T.H. Lee, S.G. Ghosh, S.D. Maharaj, D. Baboolal, arXiv preprint arXiv:1511.03976 (2015)

34. J.M. Graça, G.I. Salako, V.B. Bezerra, Int. J. Mod. Phys. D 26(10), $1750113(2017)$

35. P.S. Letelier, Phys. Rev. D 20(6), 1294 (1979)

36. P.S. Letelier, Il Nuovo Cimento B (1971-1996) 63(2), 519 (1981)

37. P.S. Letelier, Phys. Rev. D 28(10), 2414 (1983)

38. J.M. Bardeen, B. Carter, S.W. Hawking, Commun. Math. Phys. 31(2), 161 (1973)

39. J.D. Bekenstein, Phys. Rev. D 7(8), 2333 (1973)

40. S.W. Hawking, Commun. Math. Phys. 43(3), 199 (1975)

41. G.W. Gibbons, S.W. Hawking, Phys. Rev. D 15(10), 2738 (1977)

42. H. Xu, W. Xu, L. Zhao, Eur. Phys. J. C 74(9), 3074 (2014)

43. M.H. Dehghani, M. Shamirzaie, Phys. Rev. D 72(12), 124015 (2005)

44. M. Dehghani, R. Pourhasan, Phys. Rev. D 79(6), 064015 (2009)

45. D.J. Gross, E. Witten, Nucl. Phys. B 277, 1 (1986)

46. N. Dadhich, S. Hansraj, B. Chilambwe, Int. J. Mod. Phys. D 26(06), 1750056 (2017)

47. N. Dadhich, Pramana 74(6), 875 (2010)

48. G.Q. Li, Chin. Phys. C 41(4), 045103 (2017)

49. N. Dadhich, arXiv preprint arXiv:1006.0337 (2010)

50. B.P. Dolan, Class. Quantum Gravity 28(12), 125020 (2011) 